\title{
Transient Analysis Method of an Axially Magnetized Novel Passive Eddy Current Damper for Suppressing Lateral Vibrations
}

\author{
Abdelrahman I. A. Eisa $\mathbb{D}^{\mathbb{D}},{ }^{1,2}$ Li Shusen $\mathbb{D}^{1},{ }^{1}$ Zhang Yan $\mathbb{D}^{1},{ }^{1}$ and Wasim M. K. Helal $\mathbb{D}^{3,4}$ \\ ${ }^{1}$ College of Mechanical and Electrical Engineering, Northeast Forestry University, Harbin 150040, China \\ ${ }^{2}$ Department of Mechanical Engineering, Faculty of Engineering Science, University of Nyala, P.O. Box 155, Nyala, Sudan \\ ${ }^{3}$ Hainan Vocational University of Science and Technology, Haikou City, Hainan Province, China \\ ${ }^{4}$ Department of Mechanical Engineering, Faculty of Engineering, Kafrelsheikh University, Kafrelsheikh 33156, Egypt
}

Correspondence should be addressed to Li Shusen; lishusenzp@126.com

Received 20 October 2021; Accepted 20 January 2022; Published 8 February 2022

Academic Editor: Nhon Nguyen Thanh

Copyright $\odot 2022$ Abdelrahman I. A. Eisa et al. This is an open access article distributed under the Creative Commons Attribution License, which permits unrestricted use, distribution, and reproduction in any medium, provided the original work is properly cited.

\begin{abstract}
A passive eddy current damper (PECD) can be adopted to provide a damping effect and drag force in rotating structure applications. The damping effect is formed as the result of induced eddy current, while the drag force is produced as the result of the repulsive force between the same axially poled magnets. However, modeling and approximating the damping effect, repulsive force, stiffness, the magnetic flux density (MFD) distribution, and eddy current prompted in PECD is a challenging task because of the complicated structure and operational conditions. This paper presents a new method for modeling and estimating the dynamic parameters of novel PECDs such as the damping effect, repulsive force, and stiffness for suppressing vibration in rotating structure applications. This self-powered, cost-effective PECD utilizes four arc-shaped and single ring-shaped permanent magnets with a cylindrical conductor placed between the arc magnets and the ring magnet to generate a damping effect and spring. Eddy currents are generated in the cylindrical conductor as the effect of the relative motion of the ring magnet concerning the arc magnets. An accurate and transient analysis method of the proposed system is carried out by using a three-dimensional finite-element method (3D-FEM). According to the procedures applied in the FE model solution, this new numerical method is appropriate and validated for analyzing PECDs and gaining good results by configuring the conductor and permanent magnets symmetrically. Finally, this novel 3D transient analysis method presents a perfect ability and accuracy for both modeling and calculating the PECD parameters.
\end{abstract}

\section{Introduction}

To suppress the vibrations in rotating machinery, many types of dampers such as piezoelectric, viscous, and viscoelastic dampers can be utilized. Compared with these dampers, PECD has many advantages of vacuum compatibility, high thermal stability, no mechanical contact, and high reliability [1-3]. An electromotive force (EMF) of PECD is formed when a nonmagnetic conductive material is subjected to a time-varying magnetic field. This EMF generates eddy currents to circulate inside the nonmagnetic conductor resulting in a magnetic field. A force is formed due to the magnetic field made by these currents when interacting with the applied field. The schematic of this process is shown in Figure 1, and has been investigated in [4-6]. Both the force and current density is dependent on the rate of change of the applied field. As the result of the internal resistance of the conductor, the current will dissipate into heat, and the force will be damped.

During the past, a few decades back, the use of eddy currents for suppressing vibration purposes has been investigated and applied to braking systems, rotary machinery control, and structures. Eisa et al. [7] provided a numerical method for predicting the lateral vibrations; this method is an integration of both finite element method and multibody dynamics to be used for studying the damping force. MacLatchy et al. [8] established a magnetic braking experiment to calculate and measure both the magnetic forces 


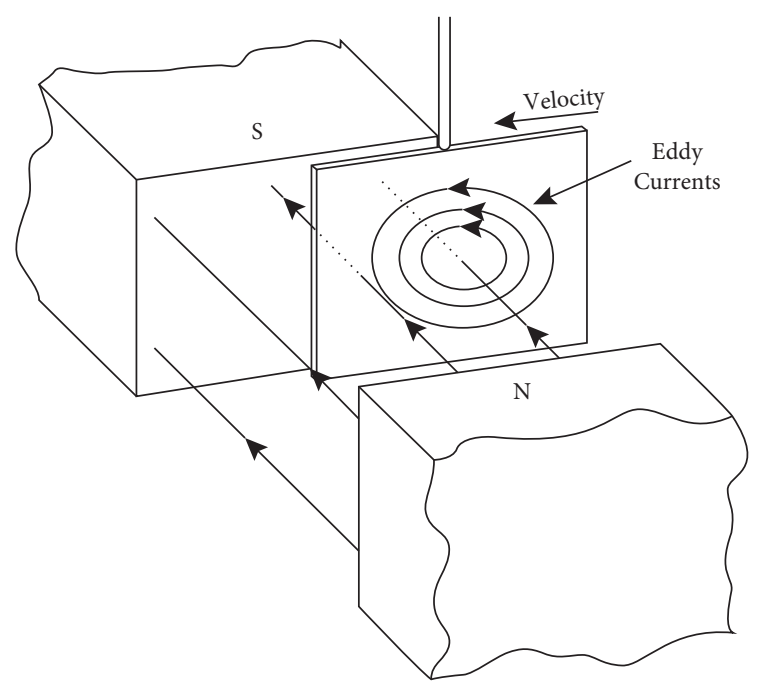

Figure 1: Configuration of conductor passing through a magnetic field and the formation of eddy currents.

and terminal velocity. The magnetic brake consisted of a magnet in a copper pipe vertically. Lee and Park [9] analyzed the eddy current in the case of the braking system based on the images method and Coulomb's law taking the boundary conditions of the system into consideration. Choi and Jang [10] studied an eddy current brake with a type of radial flux permanent magnet, and the magnetic torque was calculated analytically and tested experimentally. Cunningham [11] created a rotational eddy current damper that was similar to an eddy current brake which consisted of a rotating disk fixed on a bearing. Fredreick and Darlow [12] established ECD works under $1000 \mathrm{rpm}$, but turns into an unstable state at higher speeds, and then ECD was later modeled by Kligerman and Gottlieb [13] through a mathematical model presented to predict the damper behavior. Schmid and Varga [14] studied a vibration suppressing system using ECDs for nanotechnology devices such as a microscope. Kwak et al. [15] studied the ECD effects on cantilever beam vibration analytically and performed experimentally.

For studying PECD, it is vital to use precise modeling taking all geometric details into consideration including analytical or numerical methods. The analytical methods for modeling and design of PECD were used by several authors. Ebrahimi et al. [16] studied the feasibility of ECD automobile suspension; they used an analytical method based on elliptic integral for calculating repulsive force in ECD. Sodano et al. [17] presented the concept and model of eddy current damper and calculated the damping force induced in the proposed model based on the analytical model, and the analytical model depends on elliptic integral. Bae et al. [18] designed and tested an eddy current damping system using a permanent magnet passing inside a conductive tube and an analytical method for calculating electromagnetic force based on the elliptic integral. Ebrahimi et al. [19] configured a permanent magnet in the design of an ECD and derived an analytical method that used an elliptic integral to calculate damping force. Takayama and Kondou [20] developed a magnetic damper consisting of a combined hollow cylinder magnet and conducting disks, and an elliptic integral was used in the analytical method for calculating magnetic flux density and therefore magnetic damping force. Ebrahimi et al. [21] calculated magnetic flux density and eddy current damping force of a magnetic shock absorber based on the eddy current damping effect using an elliptic integral. Sodano and Cheah [22] presented a theoretical method for modeling eddy current damper using elliptic integrals. Analytical methods such as elliptic integrals are used for calculating magnetic force equations in radial and axial magnetization. However, the analytical methods are very complicated [23]. This complication is simplified by using numerical methods such as the finite element method.

In this work, we present a 3D transient analysis method for the gaining of the electromechanical parameters and mechanical properties of PECD for suppressing the lateral vibrations. The proposed method uses a numerical model based on the 3D FE model and then solves the Maxwell equations to gain the radial damping properties of the PECD. The solution of the 3D FE model is obtained under specific excitations and boundary conditions, and these conditions make the 3D FE model simple to implement, therefore being simple and fast to be solved. Due to the processes involved in the solution of the FE model, the proposed method is validated to PECDs employing a symmetric configuration of permanent magnets and conductors. Such type of configuration is applicable to most cases in rotor dynamic applications, especially in the field of PECDs and passive magnetic bearings PMBs. Finally, the proposed method is presenting a good accuracy for modeling the dynamic behavior of PECD and showing the perfect ability for calculating the electromechanical parameters of such systems.

\section{Methodology}

The schematic configuration of the proposed PECD is illustrated clearly in Figures 2 and 3. It consists of four arcshaped permanent magnet (PM), a single ring-shaped PM, and a cylindrical conductor. The four arc-shaped PM and the conductor are fixed to the stator, while the ring-shaped PM is fixed to the rotor and has a translational motion in a vertical direction ( $x$-axis) as shown in Figure 4(a) and a horizontal direction ( $y$-axis) as shown in Figure 4(b). For the reason of great electrical conductivity, copper is selected as the conductor material under a time-varying magnetic field which occurs by translational motion of the ring magnet, the field circulates inside the conductor, and hence an eddy current is generated. The repulsive force between the magnets has happened, and stiffness is created as well. The modeling and simulation of PECD passed through many processes using ANSYS MAXWELL. First, the 3D design of PECD was created with specific dimensions. Then, the material of each component was defined successfully. After that, a band was created around the ring magnet to perform the translational motion along $5 \mathrm{~mm}$ length and with a speed of $5 \mathrm{~m} / \mathrm{s}$. The excitations and boundary conditions were assigned to the model. Finally, a region around the PECD model was shaped, and a 3D-FEM was generated. For the 


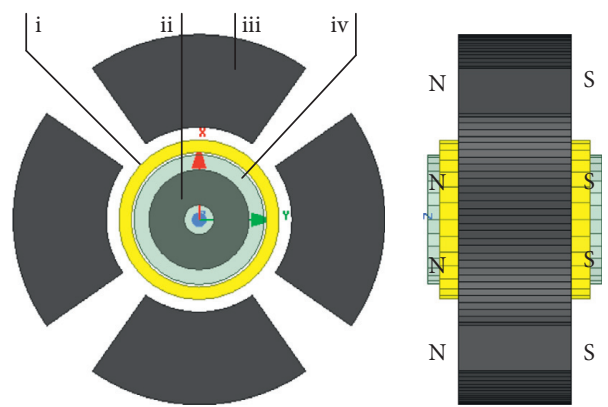

FIgURE 2: The configuration of the proposed PECD: (i) cylindrical conductor, (ii) ring-shaped PM, (iii) arc-shaped PM, and (iv) band.

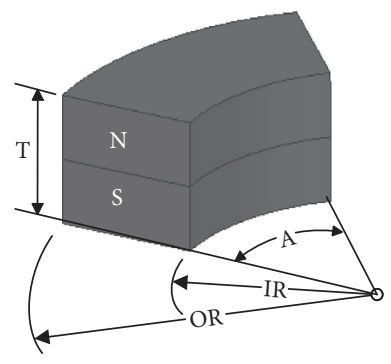

(a)

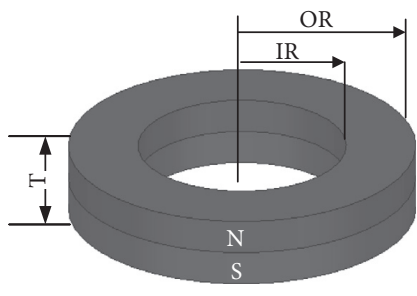

(b)

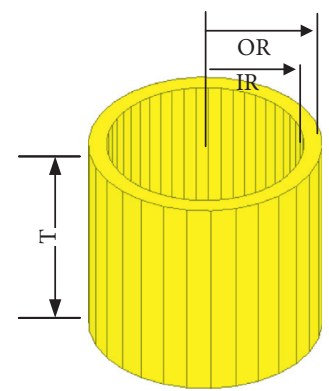

(c)

FIgure 3: The descriptions of PECD parts: (a) arc-shaped PM, (b) ring-shaped PM, and (c) the conductor.

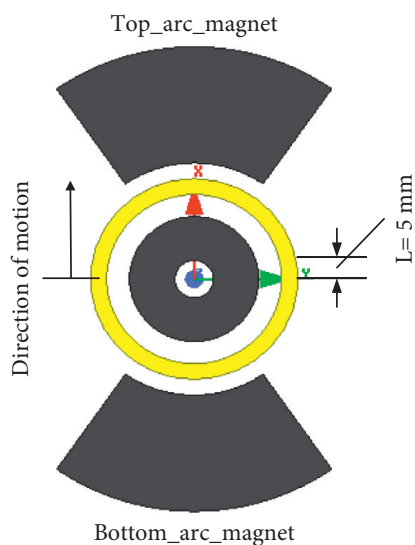

(a)

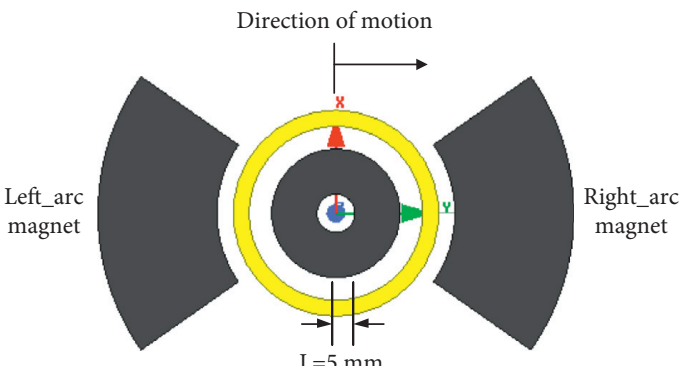

(b)

FIgUre 4: The motion description of PECD along (a) $X$-axis and (b) $Y$-axis.

proposed PECD illustrated in Figure 2, when the ring permanent magnet of velocity $v$ is moved relatively to the conductor of conductivity $\sigma$ and thickness $\delta$, the magnetic field is generated inside it. According to the configuration of PECD, the shaft deflection occurs in the $x-y$ plane. The magnetic field component along the $z$-direction does not contribute to eddy currents generation, and therefore, the electric field circulated on the conductor is strongly dependent on the radial component. The magnetic force caused by varying magnetic field induced inside the conductor can be demonstrated by the following Lorentz's force equation:

$$
F=q(E+v \times B),
$$

where $F$ is the vector of force, $q$ is the particle electric charge, $E$ is the vector of electric field intensity, $v$ is the vector of velocity, and $B$ is the vector of magnetic flux density. Because of the symmetry of the configuration existing in the PECD here, the electric field intensity is zero and can be rearranged as

$$
\frac{F}{q}=v \times B .
$$


The product of the conductivity and electric field intensity is defined as eddy current density and (2) can be rewritten as

$$
J=\sigma(v \times B) .
$$

As described in Figure 3, the parameters of the proposed PECD are given as follows: for the ring magnet, the inner radius (IR) is $5.9 \mathrm{~mm}$, outer radius (OR) $20 \mathrm{~mm}$, and thickness $(T)$ is $30 \mathrm{~mm}$. For the arc magnet, IR is $37 \mathrm{~mm}$, OR is $74 \mathrm{~mm}, T$ is $45 \mathrm{~mm}$, and arc angle $A$ is $70^{\circ}$. For the conductor, IR is $27 \mathrm{~mm}$, OR is $32 \mathrm{~mm}, T$ is $60 \mathrm{~mm}$, and conductivity is $5.5 * 10^{7}$. For the band, IR is $26 \mathrm{~mm}$ and $T$ is $70 \mathrm{~mm}$. For the rectangular region, the length is $100 \mathrm{~mm}$. Magnetization of the PMs is $995 \mathrm{kA} / \mathrm{m}$, the composition of the PMs is NdFeB 50, and MFD is 1.4 Tesla. For calculating the electromechanical parameters and mechanical properties of PECD perfectly, the proposed model was built according to the mentioned dimensions. For the reason that the ring magnet moves relative to both the conductor and arc magnet, a moving mesh in interval time is required [24]. In this case, where the arc magnet together with conductor is stationary while the ring magnet is moving, the calculation time consumption is more and therefore faster and simpler mesh type can be used. In addition, one mesh and one calculation are enough to generate the eddy current, damping effect, repulsive force, stiffness, and the magnetic flux density (MFD) distribution [25]. The tetrahedral element types were applied to the proposed model with length based type, and the maximum length was set to be $5 \mathrm{~mm}$ and the maximum elements were set to be 10000 . The mesh process is resulting in 2090 elements and 106967 nodes. The meshing of PECD is shown in Figure 5. Then, the copper was meshed with extra elements to improve the current density in the surface of the air gap. Table 1 demonstrates the mesh quality and density of PECD parts.

\section{Results and Discussion}

3.1. Air Gap Flux Distribution. Figure 6 illustrates the flux distribution of PECD on the $x-y$ plane when the ring magnet moves $5 \mathrm{~mm}$ of air gap with a speed of $5 \mathrm{~mm} / \mathrm{s}$ in a total time of 1 second along the $x$-axis, and this motion is divided into 6 equal steps with an interval of 0.2 seconds. In the air gap region, the flux concentrates as the ring magnet moves closer to the arc magnet along the $x$-axis and $y$-axis; on the other hand, when the ring magnet reaches the point of 1 second with $5 \mathrm{~mm}$, the concentration of the flux will be higher than the other point as shown in Figure 6(f) compared with (a), (b), (c), (d), and (e); therefore, as the eddy current is induced in the conductor, it generates a magnetic field opposite to the applied field produced by the magnets. The flux inside the conductor is very sparse and contributes to the Lorentz force induced on the conductor slightly.

It is necessary to study the effect of MFD B when the ring magnet moves along the $x$-axis and $y$-axis to understand the performance of MFD on the axis (along the $z$-axis). Figure 7 demonstrates the distribution of MFD B through the $z$-axis when the ring magnet moves along the $x$-axis and $y$-axis. The

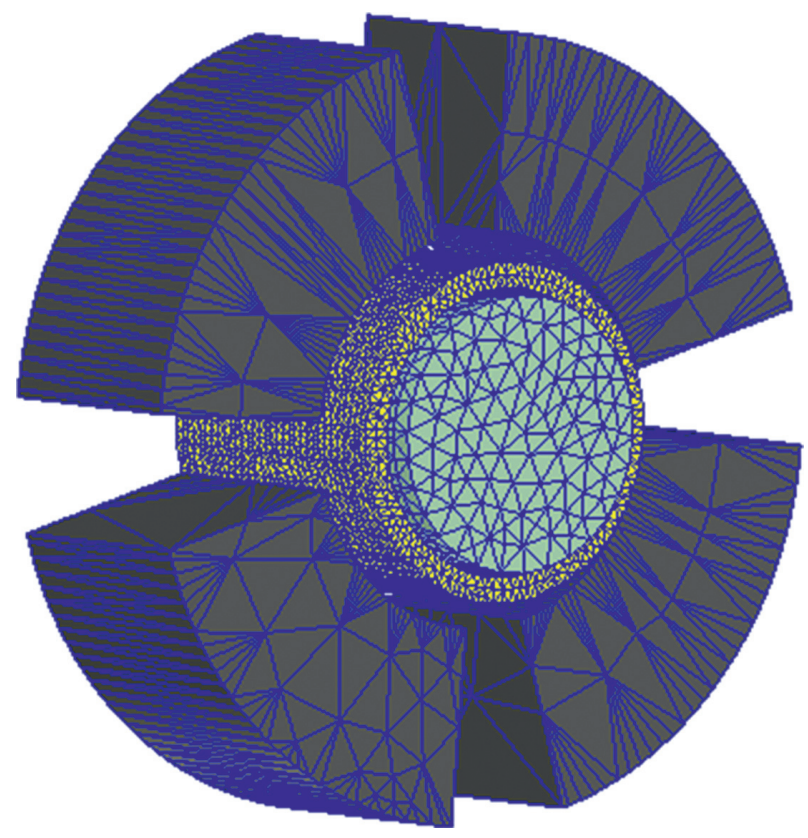

Figure 5: Meshing of PECD.

MFD is measured along a line of $70 \mathrm{~mm}$ through the $z$-axis, and this line is symmetric about the center of the ring magnet; it is noted that MFD begins with a value around $100 \mathrm{~m}$ Tesla and increases gradually when passing through the conductor and keep increasing while passing through the magnets, that is, MFD induced in the conductor is quite enough to generate eddy current which used in the damping process.

3.2. Eddy Current Distribution. Figure 8 explains eddy current distribution on the $x-y$ of PECD when the ring magnet moves from the rest $(0 \mathrm{~mm}$ at 0 seconds) to reach the final point ( $5 \mathrm{~mm}$ at 1 second) through the air gap. When the ring magnet reaches the peak position, the PECD has a larger eddy current than that at other positions because of the larger magnetic flux induced in the conductor. Moreover, it can be seen that the eddy current distributed gradually is due to the distribution of magnetic flux in the six steps mentioned. This gradual distribution in eddy current gives the system good damping.

3.3. Forces and Stiffness Distribution. In order to study the interaction forces generated in PECD, the motion of the ring magnet relative to the arc magnets was divided into two movements: the first movement is the ring magnet interacts with the left arc magnet and right arc magnet which is performed horizontally, and another movement is the ring magnet interacting with the top arc magnet and bottom arc magnet which performed vertically. These horizontal and vertical movements play a significant role in the damping process of the lateral vibration induced in the system. The transient force achieved when the ring magnet moved through the $x$-axis and $y$-axis at each time interval is presented in Figure 9. Generally, it can be observed that the 
TABLE 1: Mesh quality and density of PECD parts.

\begin{tabular}{|c|c|c|c|c|}
\hline The meshed body & Number of tetrahedral & Edge length $(\mathrm{mm})$ & Mean tetrahedral (volume $\left(\mathrm{mm}^{3}\right)$ ) & Standard deviation (volume $\left(\mathrm{mm}^{3}\right)$ ) \\
\hline Ring magnet & 313 & 30.387 & 109.067 & 126.093 \\
\hline Conductor & 63681 & 2.592 & 0.856 & 0.305 \\
\hline Left arc magnet & 729 & 37.0061 & 154.887 & 237.590 \\
\hline Top arc magnet & 692 & 38.469 & 163.169 & 249.778 \\
\hline Right arc magnet & 717 & 34.375 & 157.479 & 345.502 \\
\hline Bottom arc magnet & 807 & 37.314 & 139.917 & 227.841 \\
\hline Band & 2370 & 21.285 & 47.864 & 74.906 \\
\hline Region & 37658 & 34.663 & 850.929 & 21504.7 \\
\hline
\end{tabular}

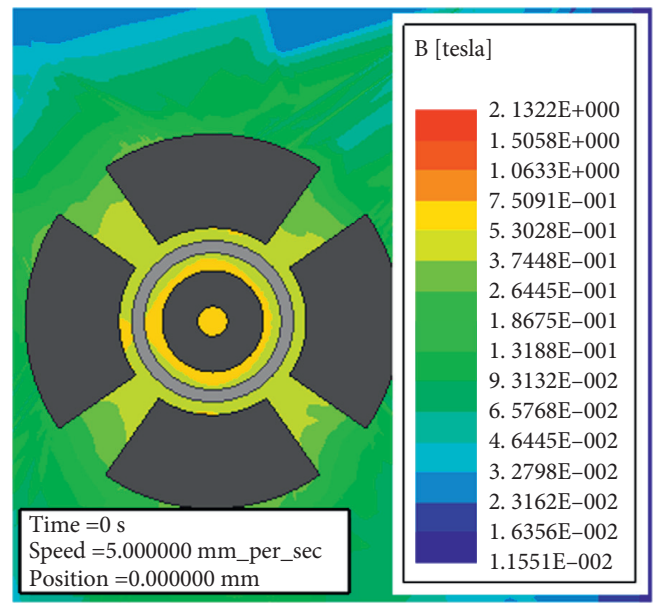

(a)

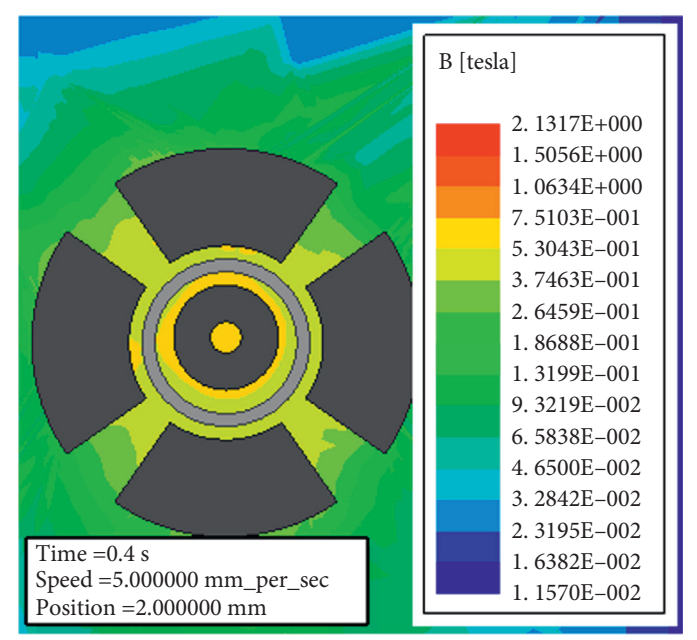

(c)

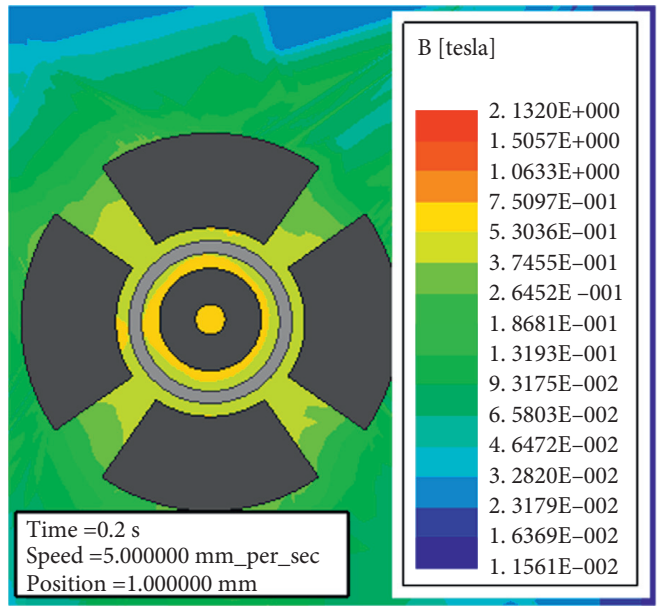

(b)

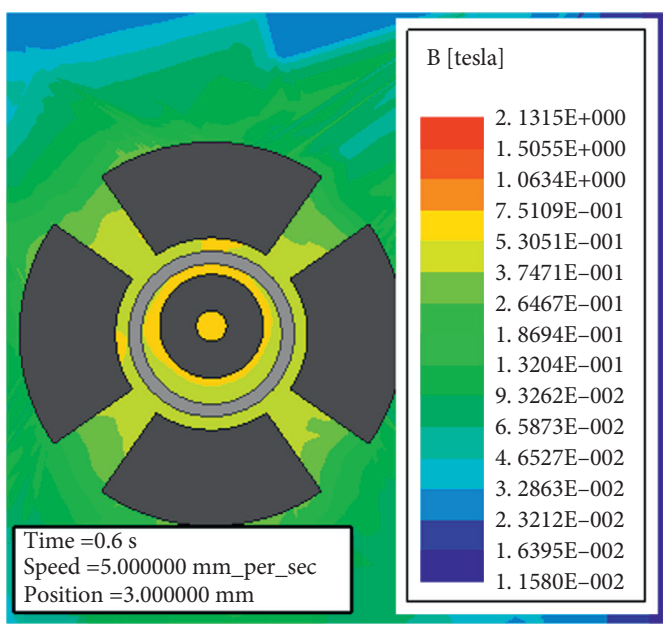

(d)

FIgURE 6: Continued. 


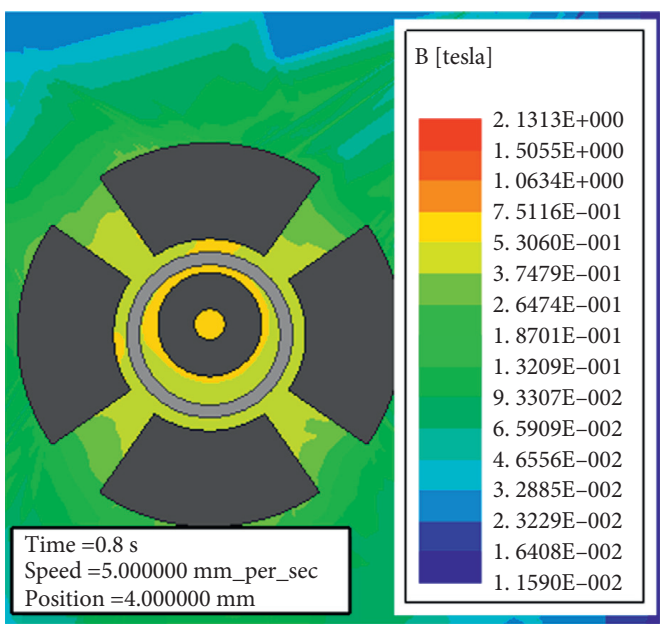

(e)

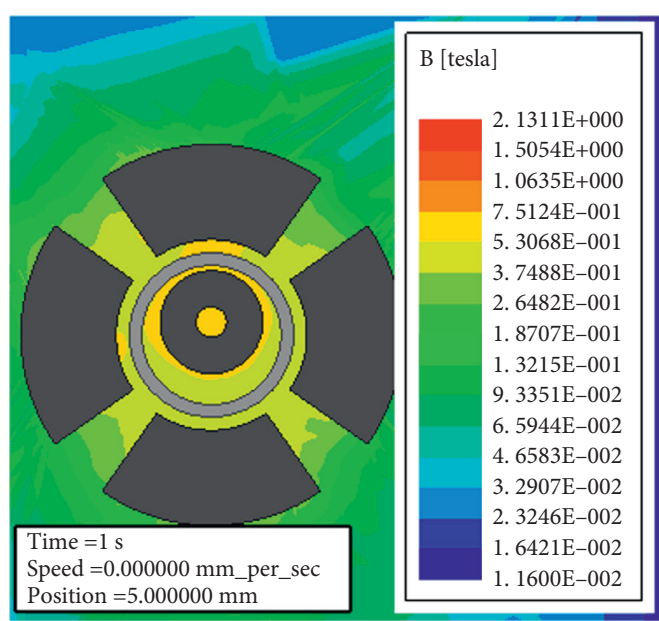

(f)

Figure 6: Flux distribution on the $x-y$ plane when the ring magnet moves $5 \mathrm{~mm}$ through air gap along the $x$-axis and reaches (a) 0 mm at 0 second; (b) $1 \mathrm{~mm}$ at 0.2 seconds; (c) $2 \mathrm{~mm}$ at 0.4 seconds; (d) $3 \mathrm{~mm}$ at 0.6 seconds; (e) $4 \mathrm{~mm}$ at 0.8 seconds; and (f) $5 \mathrm{~mm}$ at 1 second.

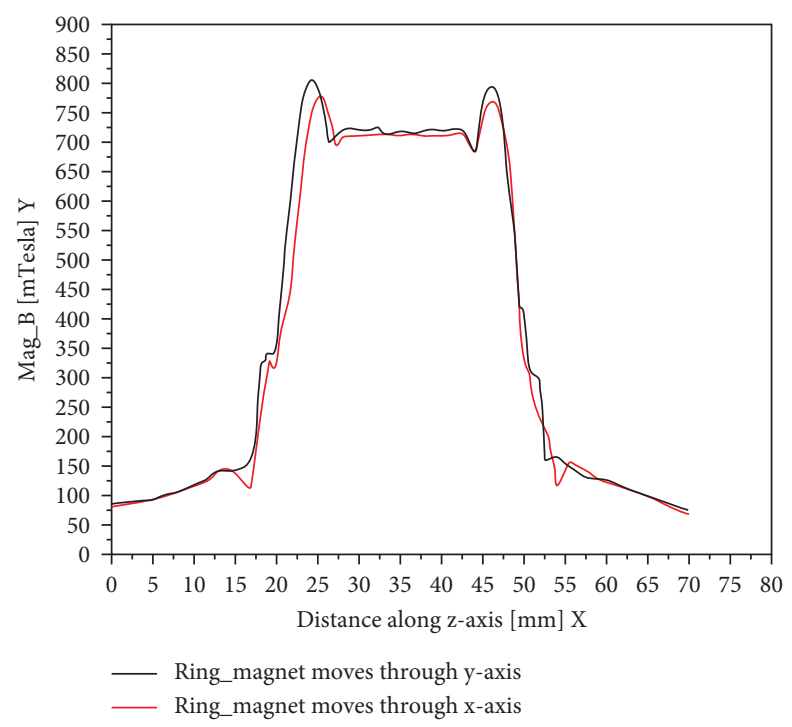

Figure 7: The MFD B distribution through the $z$-axis when ring magnet moves along the $x$-axis and $y$-axis.

force that acts through the $y$-axis is larger than those of the $x$ axis, and the acted force by the ring magnet increased as the ring magnet moves closer to the arc magnet. The interaction forces cause PECD to act as the damper and offer a restoring force to any lateral shaft motion.

For the arc magnets, as it is observed in Figures 10 and 11 , the arc magnetic force has its greater amplitude when the time reaches 1 second; at this point, the ring magnet reaches near the specified arc magnet, and the air-gap width is the smallest, but according to the physical limitation, the point at 1 second is the best width for air-gap, and the forces approach greater amplitude.

Figure 12 shows the transient forces induced in the conductor along the $x$-axis and $y$-axis. The transient force on the conductor along the $y$-axis is larger than the force induced on the conductor along the $x$-axis. Generally, the transient forces induced on the conductor are the largest as the eddy current in the conductor is the largest and that is because the ring magnet moves very close the conductor.

Figure 13 shows the magnetic stiffness that is achieved from the ring magnet versus $5 \mathrm{~mm}$ of an air gap and acted along the $x$-axis and $y$-axis; it is noted that the air gap decreases as the magnet stiffness increases; by reaching the end of the assumed air gap distance, we can achieve high stiffness, and therefore, this stiffness may apply for damping the vibration of rotating structures.

For verifying this work, a comparison with previous studies must be conducted. The structure of PECD to some extent is similar to the structure modeled in Cheah and Sodano [26] which contains two concentric ring magnets that act with inserted copper cylinder between the magnets 


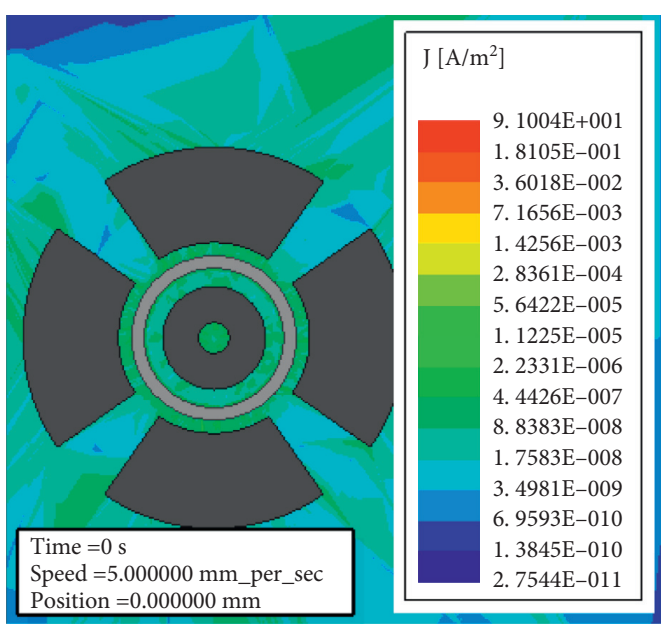

(a)

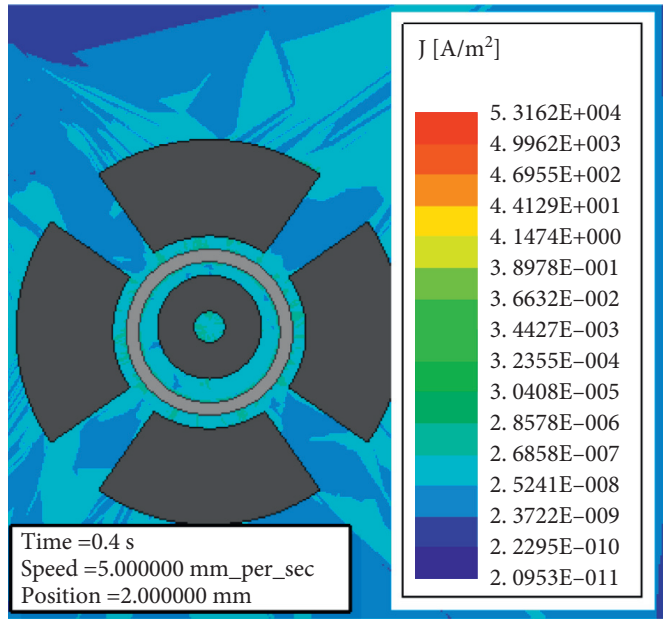

(c)

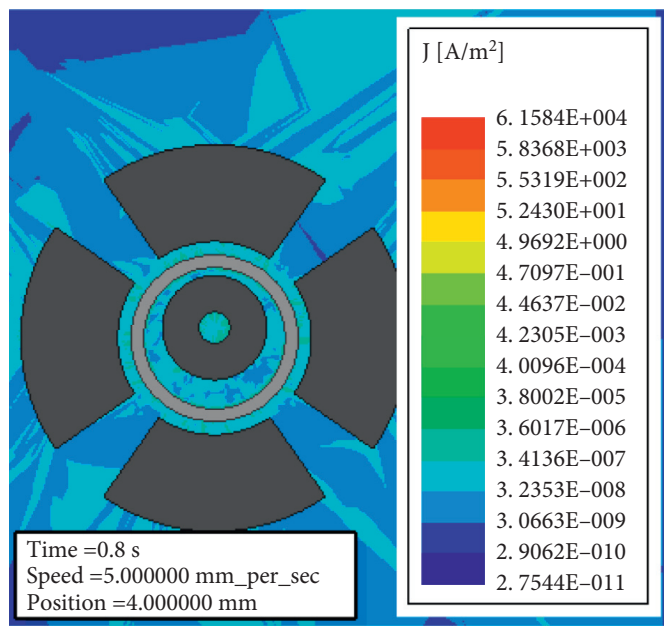

(e)

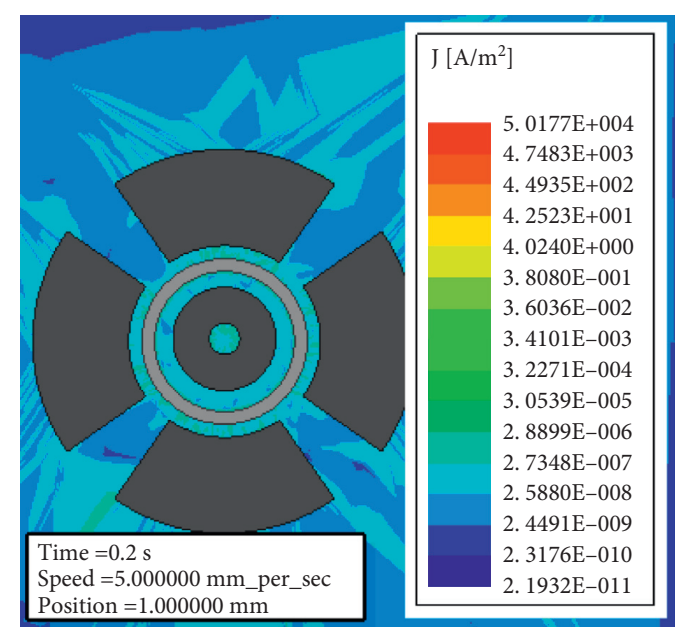

(b)

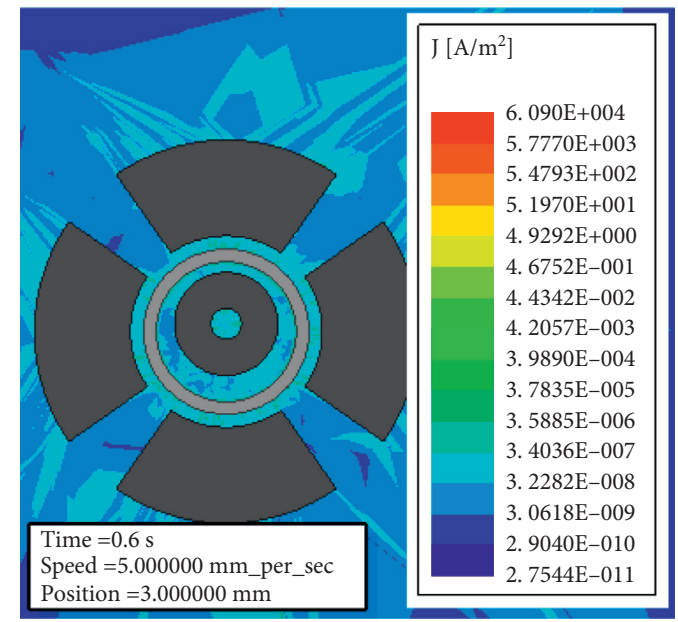

(d)

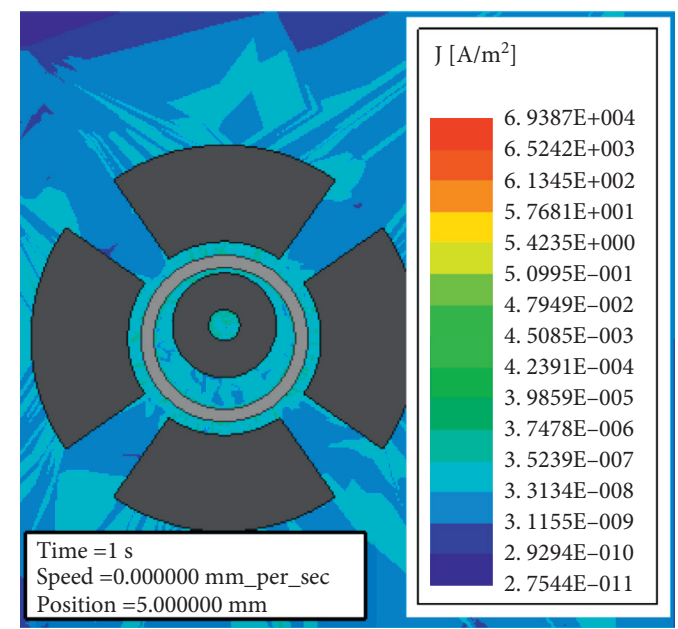

(f)

FIGURE 8: The distribution of eddy current J of PECD on the $x$ - $y$ plane when the ring magnet moves 5 mm through the air gap along the $x$ axis and reaches (a) $0 \mathrm{~mm}$ at 0 second; (b) $1 \mathrm{~mm}$ at 0.2 seconds; (c) $2 \mathrm{~mm}$ at 0.4 seconds; (d) $3 \mathrm{~mm}$ at 0.6 seconds; (e) $4 \mathrm{~mm}$ at 0.8 seconds; and (f) $5 \mathrm{~mm}$ at 1 second. 


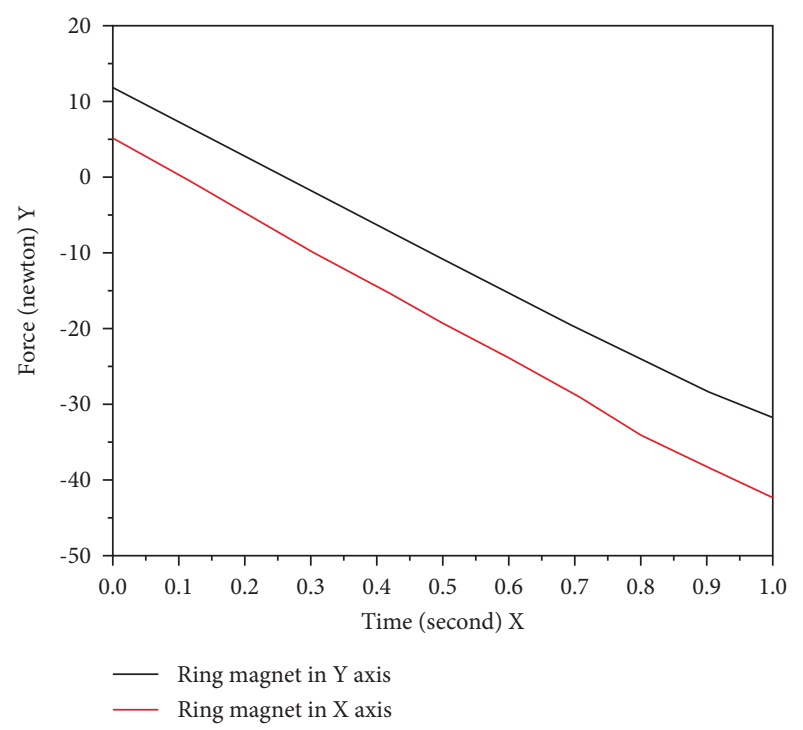

FIGURE 9: The interacted transient ring magnet force with the right, left, top, and bottom arc magnet through the $x$-axis and $y$-axis.

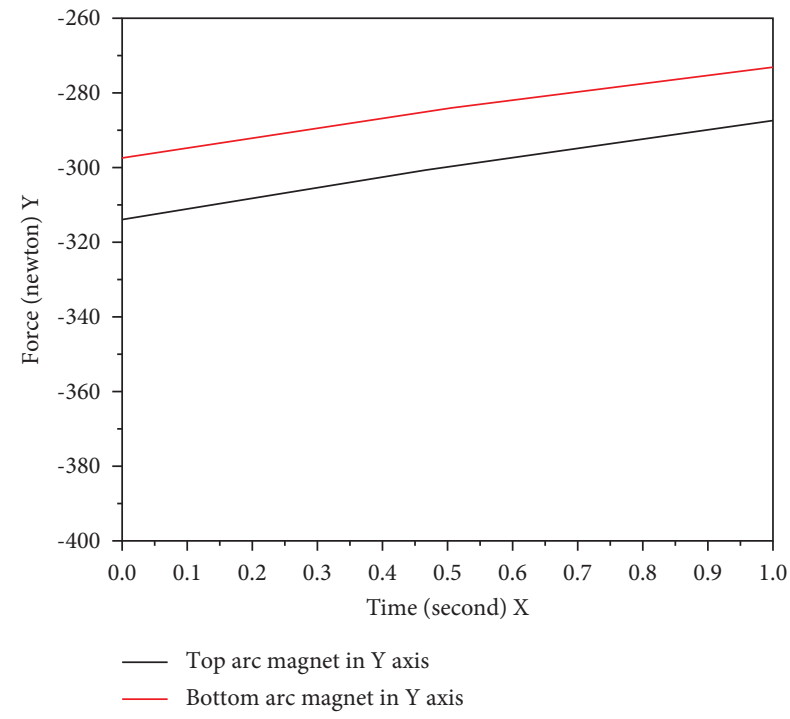

FIGURE 10: The interacted transient force of the top and bottom arc magnet with the ring magnet through the $y$-axis.

and poled axially. The stiffness between the magnets achieved in this study is quite similar to the stiffness achieved in Cheah and Sodano's work [26]. The comparison between the results is shown clearly in Figure 13. This can give an indicator that the transient analysis method used in this study is quite true. As compared this work with the previous

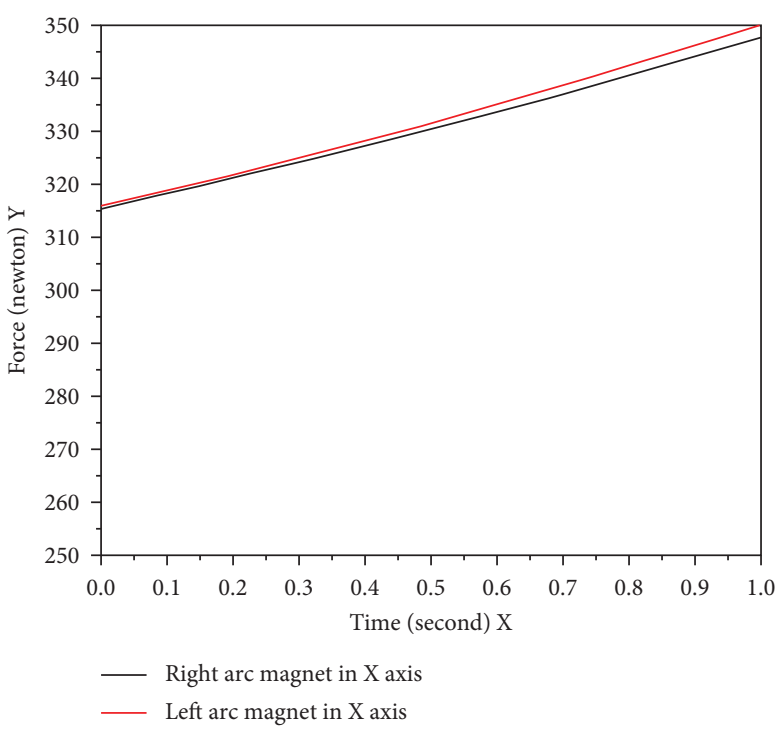

Figure 11: The interacted transient force of the left and right arc magnet with ring magnet through the $x$-axis.

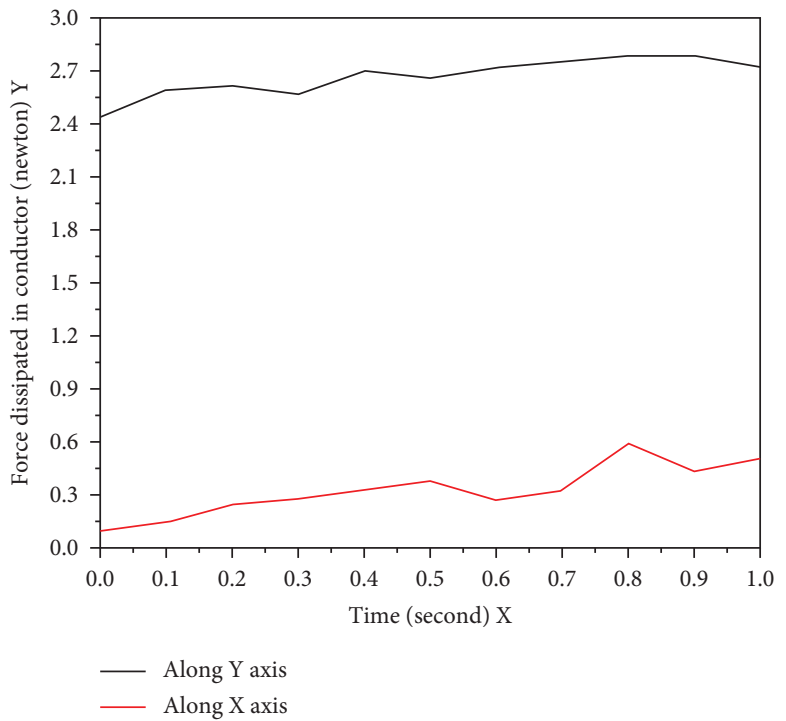

FIGURE 12: The transient forces induced on the conductor along the $x$-axis and $y$-axis.

studies which used the analytical methods, it is found that this new transient analysis method not only provided easy and accurate results for PECD parameters such as stiffness and damping effect, but it also offered deep analysis for other parameters such as repulsive force, MFD distribution, and eddy current at the same time. 


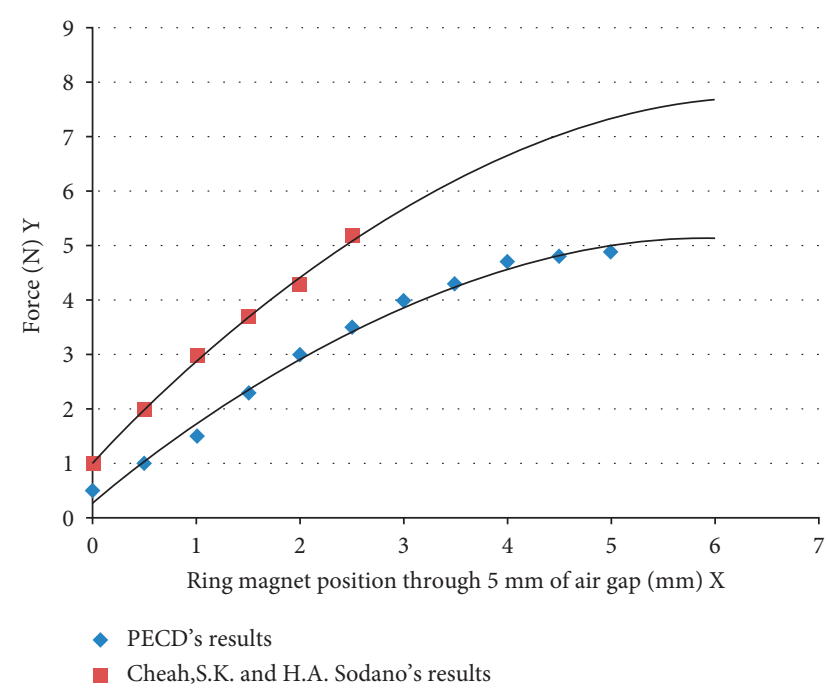

FIgURE 13: Stiffness between the ring magnet and arc magnets (PECD's results and Cheah, S.K. and H.A. Sodanos' results).

\section{Conclusions}

In the current study, a PECD was investigated by using the damping effect of eddy current. The proposed PECD used a fixed ring permanent magnet and four-arc permanent magnet working together with a conductive copper cylinder. The proposed PECD system was created by using the transient analysis method based on the motion and contribution of eddy current. The MFD, the eddy current distribution, the flux distribution, induced eddy current damping force, and the magnets' interaction forces were analytically calculated. To evaluate the design parameters, simulations were conducted. When the proposed PECD model was established, the comparison with the previous study was carried out to verify the accuracy of the analytical model. Finite element simulations have also been implemented to predict the stiffness between the ring magnet and arc magnets. The results of this work have demonstrated that PECD could be existed in rotating machinery to suppress the lateral vibrations and improve damping levels without contact in nature and no external power. Extending the PECD for greater precision could be part of future work. According to the results carried out, the proposed $3 \mathrm{D}$ transient analysis method not only can be used to analyze such PECD but also can be used to analyze the passive magnetic bearing. Besides, this proposed PECD can be used to suppress the lateral vibrations in rotating machinery.

\section{Data Availability}

The numerical simulation data files used to support the findings of this study are available from the corresponding author upon request.

\section{Conflicts of Interest}

The authors declare that there are no conflicts of interest regarding the publication of this paper.

\section{Acknowledgments}

This work was supported by the College of Mechanical and Electrical Engineering, Northeast Forestry University, Harbin 150040, China. The authors gratefully acknowledge the technical support provided by them during the course of this work. The grant fund will be under the responsibility of the corresponding author "Li Shusen."

\section{References}

[1] J.-S. Bae, M. K. Kwak, and D. J. Inman, "Vibration suppression of a cantilever beam using eddy current damper," Journal of Sound and Vibration, vol. 284, no. 3-5, pp. 805824, 2005.

[2] R.-F. Fung, J.-H. Sun, and S.-M. Hsu, "Vibration control of the rotating flexible-shaft/multi-flexible-disk system with the eddy-current damper," Journal of Vibration and Acoustics, vol. 124, no. 4, pp. 519-526, 2002.

[3] Y. Matsuzaki, T. Ikeda, A. Nae, and T. Sasaki, "Electromagnetic forces for a new vibration control system: experimental verification," Smart Materials and Structures, vol. 9, no. 2, pp. 127-131, 2000.

[4] H. D. Wiederick, N. Gauthier, D. A. Campbell, and P. Rochon, "Magnetic braking: simple theory and experiment," American Journal of Physics, vol. 55, no. 6, pp. 500-503, 1987.

[5] L. H. Cadwell, "Magnetic damping: analysis of an eddy current brake using an airtrack," American Journal of Physics, vol. 64, no. 7, pp. 917-923, 1996.

[6] J. S. Lee, "Dynamic stability of conducting beam-plates in transverse magnetic fields," Journal of Engineering Mechanics, vol. 122, no. 2, pp. 89-94, 1996.

[7] A. I. Eisa, L. Shusen, and W. M. Helal, "Study on the lateral and torsional vibration of single rotor-system using an integrated multi-body dynamics and finite element analysis," Advances in Mechanical Engineering, vol. 12, no. 10, 2020.

[8] C. S. MacLatchy, P. Backman, and L. Bogan, "A quantitative magnetic braking experiment," American Journal of Physics, vol. 61, no. 12, pp. 1096-1101, 1993.

[9] K. Lee and K. Park, "Modeling eddy currents with boundary conditions by using Coulomb's law and the method of images," IEEE Transactions on Magnetics, vol. 38, no. 2, pp. 1333-1340, 2002.

[10] J.-Y. Choi and S.-M. Jang, "Analytical magnetic torque calculations and experimental testing of radial flux permanent magnet-type eddy current brakes," Journal of Applied Physics, vol. 111, no. 7, Article ID 07E712, 2012.

[11] R. E. Cunningham, Passive Eddy-Current Damping as a Means of Vibration Control in Cryogenic Turbomachinery, National Library of Australia, Parkes, Australia, 1986.

[12] J. Frederick and M. Darlow, "Operation of an electromagnetic eddy-current damper with a supercritical shaft," Journal of Vibration and Acoustics, vol. 116, 1994.

[13] Y. Kligerman and O. Gottlieb, "Dynamics of a rotating system with a nonlinear eddy-current damper," Journal of Vibration and Acoustics, vol. 120, 1998.

[14] M. Schmid and P. Varga, "Analysis of vibration-isolating systems for scanning tunneling microscopes," Ultramicroscopy, vol. 42-44, pp. 1610-1615, 1992.

[15] M.-K. Kwak, M.-I. Lee, and S. Heo, "Vibration suppression using eddy current damper," in Proceedings of the Korean Society for Noise and Vibration Engineering Conference, Korea, August 2003. 
[16] B. Ebrahimi, M. B. Khamesee, and F. Golnaraghi, "Eddy current damper feasibility in automobile suspension: modeling, simulation and testing," Smart Materials and Structures, vol. 18, no. 1, Article ID 015017, 2008.

[17] H. A. Sodano, J.-S. Bae, D. J. Inman, and W. Keith Belvin, "Concept and model of eddy current damper for vibration suppression of a beam," Journal of Sound and Vibration, vol. 288, no. 4-5, pp. 1177-1196, 2005.

[18] J.-S. Bae, J.-H. Hwang, J.-S. Park, and D.-G. Kwag, "Modeling and experiments on eddy current damping caused by a permanent magnet in a conductive tube," Journal of $\mathrm{Me}$ chanical Science and Technology, vol. 23, no. 11, pp. 30243035, 2009.

[19] B. Ebrahimi, M. B. Khamesee, and F. Golnaraghi, "Permanent magnet configuration in design of an eddy current damper," Microsystem Technologies, vol. 16, no. 1-2, Article ID 19, 2010.

[20] Y. Takayama and T. Kondou, "Magnetic damper consisting of a combined hollow cylinder magnet and conducting disks," Journal of Vibration and Acoustics, vol. 135, no. 5, 2013.

[21] B. Ebrahimi, M. B. Khamesee, and M. F. Golnaraghi, "Design and modeling of a magnetic shock absorber based on eddy current damping effect," Journal of Sound and Vibration, vol. 315, no. 4-5, pp. 875-889, 2008.

[22] H. Sodano and S. K. Cheah, "Novel eddy current damping mechanism for passive magnetic bearings," in Proceedings of the 48th AIAA/ASME/ASCE/AHS/ASC Structures, Structural Dynamics, and Materials Conference, Honolulu, Hawaii, April 2007.

[23] M. A. Tir, S. M. Mirimani, and F. Marignetti, "A novel structure of passive magnetic bearing with axial magnetization," in Proceedings of the 5th Annual International Power Electronics, Drive Systems and Technologies Conference (PEDSTC 2014), Tehran, Iran, February 2014.

[24] J. G. Detoni, Q. Cui, N. Amati, and A. Tonoli, "Modeling and evaluation of damping coefficient of eddy current dampers in rotor dynamic applications," Journal of Sound and Vibration, vol. 373, pp. 52-65, 2016.

[25] D. Rodger, H. C. Lai, and P. J. Leonard, "A comparison of finite-element models for 3-D rotating conductors," IEEE Transactions on Magnetics, vol. 38, no. 2, pp. 537-540, 2002.

[26] S. K. Cheah and H. A. Sodano, "Novel eddy current damping mechanism for passive magnetic bearings," Journal of Vibration and Control, vol. 14, no. 11, pp. 1749-1766, 2008. 